\title{
Geometric Modeling of Medical Filtered Back-Projection Images
}

\author{
Atefeh Hasan-Zadeh \\ Fouman Faculty of Engineering, College of Engineering, University of Tehran \\ Fouman, Iran \\ Email: hasanzadeh.a [AT] ut.ac.ir
}

\begin{abstract}
This paper deals with the geometric concepts of medical imaging. The main result is proving this novel achievement that the time-frequency projections from some angles in the filtered back-projection image can be calculated by the surface area of the convex body and symplectic Holmes-Thompson volume. For this purpose, the necessary notions are introduced by the surface area of a convex body of the corresponding projective spaces, Radon and Fourier transform in integral geometry, Holmes-Thompson measure in Finsler-Minkowsi-symplectic geometry. This result leads to the other correspondence between basic notions of medical imaging such as the projections from some angles which are equally spaced, the image-frequency of these projections, the low image correctness, and the corresponding notions of Finsler geometry.
\end{abstract}

Keywords— Filtered Back-Projection, Medical Imaging, Finsler Geometry, Symplectic Geometry, Minkowski Spaces, Holmes-Thomson Volume.

\section{INTRODUCTION}

Medical imaging has led to a modern medical revolution in various ways. Many efforts have been made in this field since 1901 to date, [1-3]. The mathematical concepts of medical imaging are based on linear integrals and Fourier transforms, followed by the Radon transform, [4]. In fact, Radon transformation gives us a method for obtaining a Fourier transform of a function given by a density of its line integral.

On the other hand, many problems in geometry and physics can be easily solved by a change of viewpoint. For example, if one considers the line, or the plane, instead of the point as the basic object of geometry, the outlook changes completely. This change in viewpoint leads naturally to integral geometry. This paper is concerned with some analogy of various classical formulas from integral geometry and its relation with Finsler geometry and symplectic geometry.

This deep connection between these three geometries takes place by the type of volume named as Holmes-Thompson volume [5], which its ties to convex geometry, integral geometry, and Finsler geometry. The other advantage of working with Holmes-Thompson definition is that there is a remarkably simple formula for the Holmes-Thompson area density of a Minkowski space in terms of the Fourier transform of its norm [6-8], which is the main object in this paper.

Section 2 of this paper contains some basic concepts about Minkowski spaces, Finsler geometry and Integral geometry to cover all the key concepts in its entirety. Section 3, is about basic medical imaging concepts, too. In the sequel, the application of the triple chain of three geometries in financial markets has been proposed. The proposed methodology results in the calculation of the number of time-frequency projections from some angles in filtered backprojection image which are equally spaced in concepts of the surface area of the convex body and (symplectic) Holmes Thompson volume in notions of Fourier transform, Finsler metrics and Minkowski spaces. This fact has been proved in Theorem 1.

\section{BASIC CONCEPTS}

For an $n$-dimensional manifold $M$, let $\pi: T^{*} M \rightarrow M$ be the standard projection and let $d \pi: T\left(T^{*} M\right) \rightarrow T M$ be its differential. The canonical 1 -form $\alpha$ on $T^{*} M$ is defined by the equation $\alpha\left(V_{P_{m}}\right)=P_{m}\left(d \pi\left(V_{P_{m}}\right)\right)$, where $P_{m} \in T_{m}^{*} M$ and $V_{P_{m}} \in T_{P_{m}}\left(T^{*} M\right)$. The symplectic 2 -form is defined as $\omega=-d \alpha$. A symplectic manifold $(M, \omega)$ is a smooth manifold $M$ of even dimension $2 n$ equipped with a non-degenerate closed 2 -form $\omega$. Note that 2 -form $\omega$ on every $2 n$-vector space is non-degenerate if and only if $\omega^{n}$ is a volume form. 
Let $V$ be a vector space and $\varphi: V \rightarrow[0, \infty)$ be a norm that is smooth outside the origin. Set $L=\frac{\varphi^{2}}{2}$ and consider the exterior derivative of $L, d L$, as a map from $V \backslash\{0\}$ to $V^{*} \backslash\{0\}$. The norm $\varphi$ is said to be a Minkowski norm if $d L$ is a diffeomorphism. For any nonzero vector $v \in V$, there is an invertible linear map $D(d L)(v): T_{v} V \rightarrow T_{d L(v)} V^{*}$. In fact, using the natural identification of $T_{v} V$ with $V$, and $T_{d L(v)} V^{*}$ with $V^{*}$, it may be think of $g_{\varphi}(v):=D(d L)(v)$ as a (symmetric) bilinear form on $V: g_{\varphi}(v)\left(\omega_{1}, \omega_{2}\right):\left(D(d L)(v)\left(\omega_{1}\right)\right)\left(\omega_{2}\right)$.

The norm $g_{\varphi}$ is a Minkowski norm if and only if $g_{\varphi}$ is positive definite. When the vector $v$ belongs to the unit sphere, structure $g_{\varphi}(v)$ denoted as the osculating Euclidean structure at $v$ and the ellipsoid $E_{v}:=\left\{w \in V: g_{\varphi}(w, w)=1\right\}$, as the osculating ellipsoid at $v$. A smooth hypersurface in a finite-dimensional real vector space $V$ is said to be quadratically convex if its osculating quadrics are all ellipsoids. A vector space $V$ provided with a norm $\|\cdot\|$ such that the unit sphere is quadratically convex is called a Minkowski space.

A Finsler metric on a manifold $M$ is a continuous function defined on its tangent bundle such that $\varphi: T M \backslash\{0\} \rightarrow \mathbf{R}$ is smooth (away from the zero section) and its restriction to each tangent space is a Minkowski norm, means that the hypersurface $S_{m} M:=\left\{v \in T_{m} M: \varphi(v)=1\right\}$ is quadratically convex and encloses the origin, $(M, \varphi)$ is called a Finsler manifold.

Throughout this paper, $M$ is a compact manifold and for every $m \in M$ and any compact hypersurface $H \subset T^{*} M$, the intersection $H \cap T^{*} M$ is a convex hypersurface of $T_{m}^{*} M$ enclosing the origin and in each cotangent space $T_{m}^{*} M$ the intersection $H \cap T^{*} M$ is a quadratically convex hypersurface enclosing the origin. In this way, it can be defined as the Holmes-Thompson volume:

The Holmes-Thompson volume (1) of a $n$-dimensional Finsler manifold $(M, \varphi), \operatorname{vol}_{n}(M, \varphi)$, is the symplectic volume of its unit co-disc bundle divided by the volume of the Euclidean $n$-dimensional unit ball

$$
\operatorname{vol}_{n}(M, \varphi)=\frac{1}{\varepsilon_{n} n} \int_{S_{H}^{*} M} \alpha \wedge(d \alpha)^{n-1}
$$

where $\varepsilon_{n}$ is the volume of the Euclidean unit ball of dimension $n$.

Let $K$ is a convex body. Then it admits at least one supporting hyperplane $H$ (affine here) for any point $x$ of its boundary. The dual convex body $K^{*}$ of a given convex body $K$ is either the convex hull of the poles of the supporting hyperplanes of $H$. If the convex body $K$ be star-shaped with respect to origin in $\mathbf{R}^{n}$ and the boundary of $K$ is continuous in the sense that the Minkowski functional of $K$ defined by $\|x\|_{K}=\min \{a \geq 0: x \in a K\}$ is a continuous function on $\mathbf{R}^{n}$, then the Minkowski functional is a homogeneous function of degree 1 on $\mathbf{R}^{n}$ is strictly positive outside of the origin, and $K=\left\{x \in \mathbf{R}^{n}:\|x\|_{K} \leq 1\right\}$.

The radial function of a star body $K$ is defined by $\rho_{K}(x)=\|x\|_{K}^{-1}, x \in \mathbf{R}^{n}$. If $x \in S^{n-1}$, then $\rho_{K}(x)$ is the radius of $K$ in the direction of $x$, i.e., the distance from the origin to the boundary of $K$ in the direction of $x$.

Lemma 1. ([12]) Let $K$ be an origin-symmetric star body in $\mathbf{R}^{n}$. Then, for $0<p<n$, the function $\|\cdot\|_{K}^{-p}$ is locally integrable on $\mathbf{R}^{n}$. Also, if $f$ is a bounded integrable function on $\mathbf{R}^{n}$, then the function $\|\cdot\|_{K}^{-p} f(\cdot)$ is integrable on $\mathbf{R}^{n}$.

In this way, for $\xi \in S^{n-1}$ can be defined the parallel section function of $K$ in the direction of $\xi$ as a function on $\mathbf{R}$ given by $A_{K, \xi}(t)=v o l_{n-1}\left(K \cap\left\{\xi^{\perp}+t \xi\right\}\right)$ where $\left\{\xi^{\perp}+t \xi\right\}$ is the hyperplane perpendicular to $\xi$ at distance $t$ from the origin and can be stated as the formulation $A_{K, \xi}(t)=\int_{\langle x, \xi\rangle=t} \chi\left(\|\cdot\|_{K}\right) d x$ which $\chi\left(\|\cdot\|_{K}\right)$ is the indicator function of the body $K$. 
For $t=0$, writing the integral in the right-hand side of the function $A_{K, \xi}(t)$ in the polar coordinates of the hyperplane $\langle x, \xi\rangle=0$, result in the polar formula for the volume of central hyperplane sections

$$
\begin{aligned}
& A_{K, \xi}(0)=v o 1_{\mathrm{n}-1}\left(K \cap \xi^{\perp}\right)=\int_{\langle x, \xi\rangle=t} \chi\left(\|x\|_{K}\right) d x=\int_{S^{n-1} \cap \xi^{\perp}}\left(\int_{0}^{\infty} r^{n-2} \chi\left(r\|\theta\|_{K} d r\right)\right) d \theta \\
& =\int_{S^{n-1} \cap \xi^{\perp}}\left(\int_{0}^{\frac{1}{\|\theta\|_{K}}} r^{n-2} d r\right) d \theta=\frac{1}{n-1} \int_{S^{n-1} \cap \xi^{\perp}} \rho_{K}^{n-1}(\theta) d \theta
\end{aligned}
$$

If $\phi$ be an integrable function on $\mathbf{R}^{n}$, which is also integrable on every hyperplane, then the Radon transform of the function $\phi$ is defined as a function of $(\xi, t), \mathcal{R} \phi(\xi ; t)=\int_{\langle x, \xi\rangle=1} \phi(x) d x, \xi \in S^{n-1}, t \in \mathbf{R}$. Using (2), one can express the volume of central hyperplane sections in terms of the spherical Radon transform:

$$
A_{K, \xi}(0)=\operatorname{vol}_{\mathrm{n}-1}\left(K \cap \xi^{\perp}\right)=\frac{1}{n-1} \mathcal{R}\left(\|\cdot\|_{K}^{-n+1}\right)(\xi)
$$

for every origin-symmetric star body $K$ in $\mathbf{R}^{n}$ and every $\xi \in S^{n-1}$. Furthermore, for $(n-1)$-dimensional linear subspaces of $\mathbf{R}^{n}$, instead of $\xi^{\perp}$, the spherical Radon transform is self-dual, means that for any function $f, g \in C\left(S^{n-1}\right)$ :

$$
\int_{S^{n-1}} \mathcal{R} f(\xi) g(\xi) d \xi=\int_{S^{n-1}} f(\xi) \mathcal{R} g(\xi) d \xi
$$

There is a well-known connection between the Radon and Fourier transform:

Lemma 2. ([12]) For a fixed $\xi$, the Fourier transform of the function $g(t)=\mathcal{R} \phi(\xi ; t), t \in \mathbf{R}$ is equal to the function $z \rightarrow \hat{\phi}(\mathrm{z} \xi), z \in \mathbf{R}$.

The Fourier transform of distributions is the main tool used the proof of Theorem 1 in the sequel. For more details about the concepts mentioned in this sections can be referred to the references [13-21].

\section{RESULTS}

Medical imaging has led to a modern medical revolution in various ways. Many efforts have been made in this field since 1901 to date, [22-24].

The mathematical concepts of medical imaging are based on linear integrals and Fourier transforms, followed by the Radon transform, [25]. In fact, Radon transformation gives us a method for obtaining a Fourier transform of a function given by a density of its line integral.

Unfortunately, this transformation does not work well on non-uniform networks. For this purpose, several mathematical approaches are used, most notably:

Sample a Fourier transform on a polar coordinate grid

Interpolation of the polar grid onto a standard rectangular grid

Using the Fourier transform in polar coordinates

Localization of Radon transform

No localization of Radon transform

and some other methods in [25] and the references therein.

In all ways, the image is the filtered back-projection image using the projections from some angles which are equally spaced. Checking the sequence of the resulting images shows that the progressively improving images have more and more angular projections, until it reaches the image uses projections from the most angles. Each projection has many individual line integrals. 
In this way, to preserve the image of its projections in such a way that its properties can be preserved, the following theorem can be proved.

Theorem 1. As the notions mentioned in Methodology, the number of time-frequency projections from some angles which are equally spaced (which has been assessed by the point lies in quotation hyperplane in projective geometry) can be calculated by the surface area of the convex body and (symplectic) Holmes-Thompson volume in notions of Fourier transform, Finsler metrics and Minkowski spces.

Proof. Using of Lemma 1, Lemma 2 and self-duality of spherical Radon-transform, relation (4), it can be achieved the connection between the volume of sections of symmetric star bodies and the Fourier transform:

If $f$ be an even homogeneous function of degree $-n+1$ on $\mathbf{R}^{n}$ and continuous on the sphere $S^{n-1}$, then the Fourier transform of $f$ is even homogeneous of degree -1 and continuous on $\mathbf{R}^{n} \backslash\{0\}$ function such that, for every $\xi \in S^{n-1}$, $\mathcal{R} \phi(\xi)=\int_{S^{n-1} \cap \xi^{\perp}} f(\theta) d \theta=\frac{1}{\pi}(\hat{f}(\xi))$.

In this way, and by relation (3), it can be concluded that if $K$ is an origin-symmetric star body in $\mathbf{R}^{n}$, then the Fourier transform of the function $\|\cdot\|_{K}^{-n+1}$ is homogeneous of degree -1 function on $\mathbf{R}^{n}$ and continuous on $\mathbf{R}^{n} \backslash\{0\}$ such that, for every $\xi \in S^{n-1}, A_{K, \xi}(0)=v o 1_{\mathrm{n}-1}\left(K \cap \xi^{\perp}\right)=\frac{1}{\pi}(n-1)\left(\left(F\|\cdot\|_{K}^{-n+1}\right)(\xi)\right)$.

If $(V, \phi)$ is an $n$-dimensional Minkowski space and $N \subset V$ is an immersed submanifold of dimension $k, 1 \leq k<n$, then the formula for the Holmes-Thompson $k$-area of $N$ will be $v o l_{k}(N)=\frac{1}{\varepsilon_{k}}\left(\int_{N} \phi_{K}\right)$, where $\varepsilon_{k}$ denotes the volume of the Euclidean unit ball of dimension $k, \phi_{k}\left(v_{1} \wedge \ldots \wedge v_{k}\right):=\int_{\left(\xi_{1} \wedge \ldots \xi_{k}\right) \in S^{* k}}\left|\xi_{1} \wedge \ldots \xi_{k} \cdot v_{1} \wedge \ldots \wedge v_{k}\right| \breve{\phi}^{k}, S^{*}$ is any closed hypersurface in $V^{*} \backslash\{0\}$ that is star-shaped with respect to the origin and $\left.\breve{\phi}^{k}=-\frac{1}{4}(2 \pi)^{n-1}\left(\phi d \xi_{1} \wedge \ldots \wedge d \xi_{n}\right)\right\lrcorner X_{E}$ is the contraction of standard (distributional) Fourier transform of $\phi$ with the Euler vector field $X_{E}(\xi)=\xi$ in $V^{*}$.

In effect, there exists a smooth, translation-invariant, and possibly signed measure $\Phi_{n-k}$ on the manifold $H_{n-k}$ of $(n-k)$-flats of $V$ such that if $N \subset V$ is an immersed $k$-dimensional submanifold. Then

$$
\operatorname{vol}_{k}(N)=\frac{1}{\varepsilon_{k}}\left(\int_{\lambda \in H_{n-k}(D)} \operatorname{Cardinality}(N \cap \lambda) \Phi_{n-k}\right),[20-21]
$$

where $\varepsilon_{k}$ is the volume of the Euclidean unit ball of dimension $k$. Eventally, for a projective Finsler metric $\varphi$ on an open convex domain $D \subseteq \mathbf{R} P^{n}$ and a natural number $k, 1 \leq k \leq n-1$, equation (5) is satisfied with $D$ instead of $(V,\|\cdot\|)$, that means $\operatorname{vol}_{k}(N)=\frac{1}{\varepsilon_{k}}\left(\int_{\xi \in H_{n-k}(D)} \operatorname{Cardinality}(N \cap \lambda) \Phi_{n-k}\right)$.

In this way, geometric meaning of medical imaging result in the following correspondence:

Projections from some angles which are equally spaced $\leftrightarrow$ point lies in quotation hyperplane

Number of time-frequency projections from some angles which are equally spaced $\leftrightarrow$ surface area of convex body and (symplectic) Holmes-Thompson volume in notions of Fourier transform, Finsler metrics and Minkowski spces

Low image correctness $\leftrightarrow$ minimality of the volume in corresponding notions of geometry

In connection with the third correspondence, this should be explained that as the proof of Theorem 1, formula (5) for the Holmes-Thompson volume implies that the tangent spaces of a projective Finsler metric are hypermetric if and only if the measures $\Phi_{n-k}, k=1, \ldots, n$, are projective. Then, just like in the case of the standard Riemannian metric on $\mathbf{R} P^{n}$, 
projective subspaces are area-minimizing.

\section{CONCLUSION}

In this paper, a new geometric approach was presented to calculate the number of time-frequency projections from some angles in filtered back-projection image which are equally spaced using of the surface area of the convex body and (symplectic) Holmes-Thompson volume. In fact, the calculations were made in terms of the volume of the HolmesThomson (as the bridge between Finsler geometry, integral geometry, and symplectic geometry). The projections from some angles which are equally spaced, the number of time-frequency projections from some angles which are equally spaced, and the low image correctness was corresponded to the notions of Finsler geometry. In this way, the proposed geometric approach can provide an appropriate answer to some medical imaging issues in optimum filtered backprojection images.

\section{REFERENCES}

[1] Lipková, J., Angelikopoulos, P., Wu, S., Alberts, E., Wiestler, B., Diehl, C., Preibisch, C., Pyka, T., Combs, S.E., Hadjidoukas, P., Leemput Koumoutsakos, P., Lowengrub, J., Menze, B., "Personalized Radiotherapy Design for Glioblastoma: Integrating Mathematical Tumor Models", Multimodal Scans, and Bayesian Inference, IEEE Transactions on Medical Imaging, vol. 38, no. 8, pp. 1875 - 1884, 2019.

[2] Fioranelli, M., Sepehri, A., "A mathematical model for the virus medical imaging technique", International Journal of Geometric Methods in Modern Physics, vol. 15, no. 7, 1850121, 2018.

[3] Zayed, A.I., "A new perspective on the role of mathematics in medicine”, J Adv Res, vol. 17, pp. 49-54, 2019.

[4] Farinelli, S., "Geometric arbitrage theory and market dynamics", American Institute of Mathematical Sciences, vol. 7, no. 4, pp. 431-471, 2015.

[5] Zabreiko, P.P., Lebedev, A.V., "Banach geometry of financial market models”, Doklady Mathematics, vol. 95, no. 2, pp. 164-167, 2015.

[6] Chule, S., "Random Geometric Analysis in the Stochastic Volatility: Financial Markets States Degeneracy", Analysis and Computations Journal, Forthcoming, SSRN: https://ssrn.com/abstract=2968295, 2017.

[7] Piotrowski, E.W., Stadkowski, J., "Geometry of Financial Markets - Towards Information Theory Model of Markets”, Physica A: Statistical Mechanics and its Applications, vol. 382, no. 1, pp. 228-234, 2007.

[8] Piotrowski, E.W., Stadkowski, J., "The merchandising mathematician model: profit intensities", Physica A: Statistical Mechanics and its Applications, vol. 318, no. 3-4, pp. 496-504, 2003.

[9] Holmes, R.D., Thompson, A.C., "N-dimensional area and content in Minkowski spaces", Pacific Journal of Mathematics, vol. 85, no. 1, pp. 77-110, 1979.

[10] Paiva, J.C.A., Fernande,s E., "Fourier transforms and Holmes-Thompson volume of Finsler manifolds", International Mathematics Research Notices, vol. 19, pp. 1031-1042, 1999.

[11] Paiva, J.C.A., Thompson, A.C., Volumes on normed and Finsler spaces, Chapter book of a Sampler of RiemannFinsler Geometry, Cambridge University Press, pp. 1-48, 2004.

[12] Paiva, J.C.A., Some problems on Finsler geometry, Handbook of Differential Geometry, vol. 2, pp. 1-33, 2006.

[13] Koldobsky, A., Fourier Analysis in Convex Geometry, American Mathematical Society, vol. 116, 2006.

[14] Benjancu, A.A., Finsler geometry and applications, Ellis Horwood, 1996.

[15] Gelfand, I.M., Smirnov, M., "Lagrangians satisfying Crofton formulas, Radon transforms, and nonlocal differentials", Advances in Mathematics, vol. 109, no. 2, pp. 188-227, 1994.

[16] Gromov, M., "Filling Riemannian manifolds, Journal of Differential Geometry”, vol. 18, no. 1, pp. 1-147, 1998.

[17] Rudin, W., Functional Analysis, Mc Graw-Hil. New York, 1973.

[18] McDuff, D., Salamon, D., Introduction to symplectic topology, Oxford Mathematical Monographs, Clarendon Press, 1998.

[19]Dym, H., McKean, H.P., Fourier series and Integrals, Academic Press, New York, 1997.

[20] Schneider, R., “On integral geometry in projective Finsler spaces”, Izvestiya Natsional'noĭ Akademii Nauk Armenii. Matematika, vol. 37, pp. 34-51, 2002.

[21] Schneider, R., Wieacker, J.A., "Integral geometry in Minkowski spaces”, Advances in Mathematics, vol. 129, no. 2, pp. 222-260, 1997.

[22] Paiva, J.C.A, Fernandes, E., "Crofton formulas in Projective Finsler spaces”, Electronic Research Announcements of the American Mathematical Society, vol. 4, pp. 91-100, 1998.

[23] Olson, T., De Stefano, J., "Wavelet localization of the radon transform", IEEE Signal Process, vol. 42, no. 8, pp. 2055-2067, 1994.

[24] Olson, T., "Optimal time-frequency projections for localized tomography”, Ann. Biomed, Eng., vol. 23, pp. 622-636, 1995.

[25] Stanton, A., "Wilhelm Conrad Röntgen on a new kind of rays: translation of a paper read before the Würzburg Physical and Medical Society”, Nature, vol. 253, pp. 274-276, 1895.

[26] Olson, T., Applied Fourier Analysis: From Signal Processing to Medical Imaging, Springer, 2017. 